Cahiers de la recherche sur les droits

Cahiers

Fon les Droits fondamentaux

$8 \mid 2010$

La liberté d'expression

\title{
La liberté de pensée et d'expression en Turquie
}

Ibrahim Ö. Kaboğlu

\section{OpenEdition}

Journals

Édition électronique

URL : https://journals.openedition.org/crdf/6137

DOI : $10.4000 /$ crdf.6137

ISSN : 2264-1246

Éditeur

Presses universitaires de Caen

Édition imprimée

Date de publication : 20 décembre 2010

Pagination : 33-44

ISBN : 978-2-84133-367-7

ISSN : 1634-8842

Référence électronique

Ibrahim Ö. Kaboğlu, «La liberté de pensée et d'expression en Turquie », Cahiers de la recherche sur les droits fondamentaux [En ligne], 8 | 2010, mis en ligne le 08 octobre 2020, consulté le 14 novembre 2022. URL : http://journals.openedition.org/crdf/6137; DOI : https://doi.org/10.4000/crdf.6137 


\title{
La liberté de pensée et d'expression en Turquie
}

\author{
Ibrahim Ö. KABOĞLU \\ Professeur de droit constitutionnel à I'Université de Marmara (Istanbul)
}

\author{
I. L'évolution de la liberté d'expression \\ A. Les réformes constitutionnelles: \\ vers le droit européen et international des Droits de l'homme \\ 1. La consolidation des libertés constitutionnelles \\ 2. La juridictionnalisation des Droits de l'homme au niveau européen \\ 3. Le processus d'internationalisation des Droits de l'homme \\ $B$. Les réformes législatives \\ II. L'évolution jurisprudentielle et la pratique \\ A. La pratique de la Cour européenne des Droits de l'homme \\ B. L'évolution de la jurisprudence nationale \\ 1. La CEDH comme norme secondaire \\ 2. L'application de l'article 10 de la CEDH \\ 3. Le nouveau Code pénal et l'application du critère de danger clair et proche
}

Les réformes constitutionnelles et législatives réalisées en Turquie sont parfaitement en phase avec l'aspect évolutif de la liberté de pensée et d'expression (I). Et si la jurisprudence et la pratique en la matière démontrent des avancées, de nombreuses difficultés perdurent (II). L'évaluation de l'état de cette liberté ne peut être comprise que dans le contexte politico-social du pays, toujours en transition, marqué par le conflit des valeurs, malgré une évolution démocratique - certes difficile mais irréversible.

En Turquie, l'utilisation du terme « liberté de pensée » (düşünce özgürlüğ̈̈) est plus fréquente que l'usage de «liberté d'expression» (ifade özgürlüğ̈̈, anlatım özgürlüğü, açıklama özgürlüğ̈̈), en fait comme en droit. Pour cette raison, l'appellation de "liberté d'expression des idées» est très souvent privilégiée afin de refléter la liaison inséparable entre les deux libertés. En réalité, la liberté d'expression, sans faire référence à la liberté de pensée, a entraîné des conséquences restrictives sur le contenu de cette liberté qui s'appuie essentiellement sur les idées et les opinions ${ }^{1}$.

Au niveau des textes, il convient de rappeler que dans la Constitution précédente, entrée en vigueur le 9 juillet 1961, cette liberté n'était énoncée que dans l'article unique intitulé « la liberté de pensée » : «Chacun jouit de la liberté de pensée et d'opinion; il peut exprimer et publier individuellement ou bien collectivement sa pensée et ses opinions par paroles, écrits, illustrations ou par

1. Pour l'utilisation du titre «Les libertés de la pensée », voir F. Sudre, J.-P. Marguénaud, J. Andriantsimbazovina, A. Gouttenoire et M. Levinet, Les grands arrêts de la Cour européenne des Droits de l'homme, $5{ }^{\mathrm{e}}$ édition, Paris, PUF (Thémis droit), 2009, p. 567 sq. 
tout autre moyen. Personne ne peut être astreint à divulguer sa pensée et ses opinions. » (art. 19). En revanche, la Constitution en vigueur (qui date du 7 novembre 1982) a aménagé la liberté en cause dans deux articles séparés: «la liberté de pensée et d'opinion » (art. 25) et «la liberté d'expression et de propagation de la pensée» (art. 26).

L'article 25, qui ne prévoit aucun motif de limitation, met l'accent sur l'aspect négatif de la liberté de pensée et ses garanties. Après avoir posé le principe de la liberté d'expression, l'article 26, quant à lui, prévoit que celle-ci comprend également la faculté de recevoir et de communiquer des idées ou des informations en dehors de toute intervention des autorités officielles.

En ce qui concerne l'exercice de ces libertés, plusieurs motifs spécifiques de limitation ont été introduits dans le paragraphe 2 de l'article 26 , ces raisons dépassant la liste dressée par le paragraphe 2 de l'article 10 de la Convention européenne des Droits de l'homme $(\mathrm{CEDH})^{2}$.

$\mathrm{Au}$ niveau législatif, il convient de signaler tout d'abord le Code pénal et la loi de lutte contre le terrorisme qui contenaient les dispositions introduisant d'une façon implicite le délit d'opinion. Ensuite, la loi sur les partis politiques contient également des dispositions permettant de porter atteinte à la liberté d'expression.

Ceci dit, sur le plan juridique, le régime de la liberté d'expression peut être abordé en deux temps : la période qui précède les réformes réalisées au cours de la dernière décennie et celle qui suit les réformes. Une telle distinction peut être aussi opérée en ce qui concerne la pratique ${ }^{3}$.

- Plusieurs articles du Code pénal turc, qui réprimaient surtout certains types de propagande politique et religieuse, ont été abrogés en 1991 par la loi de lutte contre le terrorisme ${ }^{4}$. Toutefois, celle-ci avait introduit un délit de propagande dans son article 8 . La protection de «l'intégrité indivisible de l'État avec son territoire et sa nation» constituait le centre de gravité du délit d'opinion. Le cadre constitutionnel d'un tel délit était assuré par l'article 14 de la Constitution intitulé «non-abus des droits et des libertés fondamentaux ».

- La pratique en était la preuve : «À Ankara, Istanbul, Izmir et Diyarbakır, les CSE ont poursuivi et condamné des journalistes, écrivains, éditeurs, universitaires, syndicalistes, politiciens, avocats, militants des Droits de l'homme pour avoir commis des infractions relevant soit de l'article 312 du Code pénal turc, soit de l'article 8 de la loi de lutte contre le terrorisme. ${ }^{5}$ »

- La pratique ne se limitait pas aux délits d'opinion individuelle, elle condamnait également «l'utilisation collective de la liberté d'expression » par le biais des partis politiques.

Les premiers arrêts de la Cour européenne des Droits de l'homme (Cour EDH) en la matière ont confirmé cette approche ${ }^{6}$. La différenciation jurisprudentielle entre le juge national et le juge européen due à cette pratique fut assez visible à partir des premiers arrêts rendus par la Cour EDH en ce qui concerne les articles 10 et 11 de la Convention ${ }^{7}$. Comme nous avons déjà eu l'occasion de l'évoquer $^{8}$ : «La jurisprudence nationale prend en principe en considération "l'acte fait" (l'acte lui-même) à savoir les discours, les tracts (ou communiqués) rédigés, les paroles prononcées ou diffusées. Quant aux instances de Strasbourg, celles-ci mettent plutôt l'accent sur "l'impact" de l'acte commis...» «Les cours nationales dépendent du principe de la légalité stricto sensu alors que les instances de Strasbourg dépassent le seuil de la légalité dans la mesure où l'acte réprimé n'est pas lié à la violence ${ }^{9} \ldots{ }^{\prime}$

Dans quelles mesures ces remarques sont-elles encore valables en 2010 ?

La nécessité d'une réforme juridique au niveau constitutionnel et au niveau légal a été soulignée, mais «les devoirs et des responsabilités » inclus dans l'exercice de ces libertés ne devraient pas non plus être négligés par les titulaires de la liberté d'expression ${ }^{10}$.

En effet, les réformes constitutionnelles réalisées en 2001 et 2004 ont été mises en pratique par plusieurs lois qui s'appelaient les lois d' « harmonisation ». Il convient donc d'étudier l'évolution jurisprudentielle sous l'optique de la transformation du droit positif. Toutefois, la problématique de la liberté d'expression en Turquie doit être prise en compte dans une perspective plus large.

2. Pour le détail, voir I. Ö. Kaboğlu, «La liberté d'expression en Turquie», Revue trimestrielle des Droits de l’homme, $\mathrm{n}^{\circ} 38,1999, \mathrm{p} .255-256$.

3. Les développements suivants renvoient à des passages de notre article rédigé avant les réformes de la première décennie des années 2000 . I. Ö. Kaboğlu, «La liberté d'expression... », p. 253-276.

4. Loi ${ }^{\circ} 3713$, du 12 avril 1991, Terörle Mücadele Kanunu.

5. I. Ö. Kaboğlu, «La liberté d'expression... », p. 263.

6. La Turquie a reconnu le recours individuel devant la Cour EDH en 1987 et la compétence obligatoire de la Cour EDH en 1990.

7. L'affaire Zana (25 novembre 1997) et l'affaire Íncal (9 juin 1998) c. Turquie sont les premiers arrêts de la Cour EDH relatifs à l'article 10, et les affaires Parti communiste unifié de Turquie (30 janvier 1998) et Parti socialiste c. Turquie (25 mai 1998) sont les premiers arrêts dans le cadre de l'utilisation collective de la liberté d'expression.

8. I. Ö. Kaboğlu, «La liberté d'expression... », p. 273-274.

9. Il s'agissait également de divergences en ce qui concerne «l'utilisation collective » de la liberté d'expression : «Les passages sur lesquels l'accent est mis par la Cour constitutionnelle et la Cour européenne sont bien différents [...] Pour la Cour européenne, l'essentiel, c'est la démocratie; pour la Cour constitutionnelle, l'essentiel, c'est l'intégrité de l’État. » I. Ö. Kaboğlu, «La liberté d'expression... », p. 273.

10. «Pour les intellectuels d'origine kurde, la responsabilité impose de ne pas mêler "l'éloge de la violence" et la liberté d'expression. En effet, c'est seulement le débat libre qui peut contribuer à la cause kurde; sinon la violence ne fait que "tuer".»

"Pour les intellectuels partisans de la "politique officielle”, la responsabilité impose de ne pas sacraliser l'État: des restrictions à la liberté d'expression afin de préserver l'État unitaire peuvent exercer les effets inverses... Car les restrictions excessives destinées à la protection de l'intégrité indivisible de l'État “tuent” également la liberté d'expression. » I. Ö. Kaboğlu, «La liberté d'expression... », p. 275-276. 


\section{L'évolution de la liberté d'expression}

Les facteurs d'évolution de la liberté d'expression sont à la fois constitutionnels et législatifs : les réformes constitutionnelles successives réalisées dans l'objectif de promouvoir les Droits de l'homme en général (A) et les réformes législatives mettant en pratique les dispositions constitutionnelles révisées (B).

\section{A. Les réformes constitutionnelles: vers le droit européen et international des Droits de l'homme}

Les espaces constitutionnel, européen, mais également international, se complètent afin de mieux reconnaître, protéger et promouvoir les Droits de l'homme.

\section{La consolidation des libertés constitutionnelles}

La Constitution de 1961 représente le point culminant des garanties constitutionnelles en matière de droits et de libertés. Cela peut être conçu comme la constitutionnalisation des Droits de l'homme (si l'on prend en compte la valeur normative de la loi fondamentale). Elle est d'abord marquée par une approche sociale et dynamique des Droits de l'homme, alors que la loi fondamentale de 1924 était limitée à la définition du droit naturel. Ensuite, la Constitution de 1961, après avoir prévu le principe de légalité dans le respect de la Constitution, interdisait au législateur de porter atteinte à l'essence des droits, celle-ci étant considérée comme noyau intangible des droits et des libertés. En outre, un système de « limitation différenciée » d'après le caractère de la liberté en cause ne pouvait être mis en pratique que conformément à l'esprit et à la lettre de la Constitution. Enfin, l'étendue des droits et des libertés était systématisée suivant une triple classification : droits individuels, droits sociaux et économiques, et droits politiques. La normativité des Droits de l'homme a d'ailleurs été assurée par la Cour constitutionnelle, qui constitue également une protection jurisprudentielle des Droits de l'homme. Elle a utilisé divers procédés de matérialisation des normes comme la méthode évolutive de la Constitution, celle de « la détermination exacte du sens propre de la Constitution » ou «l'enrichissement de la Constitution ${ }^{11}$.

La Constitution de 1982, tout en maintenant l'aspect systématique des droits et des libertés tel qu'il a été reconnu dans la norme fondamentale précédente, renversa le système de garantie et de restriction en établissant une protection différenciée et affaiblie, et une limitation généralisée. "L'essence de la liberté» comme critère de garantie a ainsi été remplacée par les «nécessités d'une société démocratique», dans l'optique de la $\mathrm{CEDH}$. Ensuite, une clause générale des restrictions a été introduite alors que des raisons spécifiques de limitations étaient prévues pour la plupart des libertés constitutionnelles (art. 13). En bref, la Constitution était plutôt conçue comme instrument de stabilité et de l'autorité, mais pas comme technique de liberté.

Au cours du processus d'une restauration de l'État de droit ${ }^{12}$, la nouvelle version de l'article 13 de la Constitution rédigée en 2001, qui a supprimé la clause générale de restriction et renforcé les critères de garanties, a constitué l'axe majeur des réformes du droit constitutionnel des libertés. Ces quatre critères - qui peuvent d'ailleurs être aussi bien qualifiés de garanties que de limites aux restrictions - sont la société démocratique, l'essence du droit, la proportionnalité et la République laïque. En même temps, l'article 14 a été rédigé en référence à l'article 17 de la CEDH afin de consolider la liberté d'expression $^{13}$.

D'autre part, les réformes constitutionnelles, entamées en 1987, élargies en 1995 et approfondies en 2001, ont été mises en pratique par huit lois d'harmonisation et de nombreuses nouvelles lois. Plusieurs programmes de formation aux Droits de l'homme destinés aux fonctionnaires $-\mathrm{y}$ compris la quasi-totalité des juges et des procureurs - ont également été réalisés. Cependant, la restauration de l'État de droit reste inachevée, autant sur le plan normatif que sur le plan institutionnel. Les problèmes qui perdurent dans le domaine des Droits de l'homme peuvent être partiellement expliqués par le manque de réformes institutionnelles fondamentales. Toutefois, le non-respect et la violation des Droits de l'homme dépendent, dans une large mesure, de la volonté des acteurs politiques et de la mentalité des fonctionnaires $-\mathrm{y}$ compris juges et procureurs ${ }^{14}$.

\section{La juridictionnalisation des Droits de l'homme au niveau européen ${ }^{15}$}

La Turquie est l'un des premiers États contractants à avoir ratifié la CEDH, le 18 mai 1954. Cette ouverture vers la sphère européenne peut être considérée comme la

11. Pour davantage de détails, voir le «Rapport général » d'Erol Cansel dans La hiérarchie des normes constitutionnelles et sa fonction dans la protection des droits fondamentaux, VIII Conférence des Cours constitutionnelles européennes (Ankara, 7-10 mai 1990), Strasbourg - Kehl, Engel, 1990, p. 151-200 (publication en quatre langues : turc, allemand, français, anglais).

12. «La restauration de l'État de droit» est utilisée pour les réformes constitutionnelles réalisées de 1987 à 2004. Voir I. Ö. Kaboğlu, «La Turquie à l'épreuve de l'État de droit ", Démocratie et liberté: tension, dialogue, confrontation. Mélanges en l'honneur de Slobodan Milacic, Bruxelles, Bruylant, 2007, p. 117-128.

13. La rédaction de l'article 14 relatif au non-abus des droits et libertés fondamentaux a également introduit, d'une façon indirecte, la formule de la Constitution précédente, énoncée dans son article 2: «La République démocratique et laïque fondée sur les Droits de l'homme (l'article 14, modifié en 2001).

14. Pour le détail des réformes, voir I. Ö. Kaboğlu, «De la réforme constitutionnelle en Turquie », Les chroniques de l’OMIJ, n 1 , janvier 2004, p. 9-31; "Vers le droit constitutionnel des libertés ", Essays in honour of Georgios I. Kassimatis, Athènes - Berlin - Bruxelles, Sakkoulas - Berliner WissenschaftsVerlag - Bruylant, 2004, p. 939-955.

15. I. Ö. Kaboğlu, «L'évolution des Droits de l'homme et de la démocratie en Turquie depuis un demi-siècle», Bulletin des Droits de l'homme, nº 11-12, 2005, p. 112-122. Voir aussi T. W. Smith, «Leveraging norms : the ECHR and Turkey’s Human Rights reforms », in Human Rights in Turkey, Z. F. Kabasakal Arat (éd.), préface de R. Falk, Philadelphie, University of Pennsylvania Press, 2007, p. 262-274. 
conséquence naturelle de la présence de la Turquie pendant l'élaboration de la CEDH et de l'instauration de la démocratie à partir de 1946. La CEDH a donc été mise en vigueur sous la Constitution de 1924. Par rapport à la CEDH, la Constitution de 1961 représentait le «standard maximum» alors que l'inspiration de la CEDH par la Constitution de 1982 était plutôt limitée du point de vue des critères de restrictions. C'est à partir de 1987 que le contraste constitutionnel s'atténue vis-à-vis de la CEDH et ce sur deux plans: d'une part, la reconnaissance du recours individuel (1987) et de la compétence obligatoire de la Cour (1990) a eu un effet «juridictionnel» sur le droit national au cours des années 1990. D'autre part, c'est la CEDH qui constituait l'axe de la plupart des révisions constitutionnelles, effectuées d'une façon graduelle. On pourrait même avancer que la CEDH fut une sorte de "dénominateur commun » des deux Constitutions, celle de 1961 et celle de 1982, au cours du processus de la restauration de l'État de droit.

Du point de vue de l'effet des arrêts de la Cour EDH, il faut d'abord signaler que la réouverture d'un procès à la suite de la constatation de la violation de la CEDH a été reconnue en 2002 par une loi qui figure parmi les mesures d'harmonisation visant à éliminer les dispositions antilibérales dans la législation promulguée sous le régime exceptionnel du début des années 1980. Mais le poids européen sur le processus de restauration ne se limite pas au seul Conseil de l'Europe, la candidature à l'Union européenne pouvant également être considérée comme un facteur d'accélération des réformes. Dans la pratique, il convient de souligner l'effet jurisprudentiel de la Cour européenne sur l'évolution vers un « régime véritablement démocratique», d'une part par ses arrêts concernant les partis politiques dans lesquels la violation de l'article 11 a été constatée et, d'autre part, par ses arrêts calqués sur les arrêts de la Cour constitutionnelle d'Ankara ${ }^{16}$. Le développement jurisprudentiel n'est donc pas limité par l'effet sur le droit national: il implique une interaction entre la Cour européenne et les cours nationales. Au fur et à mesure, les juges de la première instance se réfèrent et citent plus souvent la jurisprudence de la Cour de Strasbourg. Une telle tendance serait accentuée par le processus d'internationalisation.

\section{Le processus d'internationalisation des Droits de l'homme}

La place des instruments internationaux en droit interne a suscité, la plupart du temps, des divergences sur le plan doctrinal comme jurisprudentiel. D'après la Constitution, les traités dûment en vigueur ont force de loi. Ils ne peuvent être soumis au contrôle de la constitutionnalité (art. 90, dernier alinéa). À cette disposition, s’ajouta la phrase suivante lors de la modification réalisée en 2004: «En cas de conflit entre une loi et les dispositions d'un traité international relatives aux Droits de l'homme, les dispositions du traité international seront appliquées. »

Cette disposition constitue une nouveauté primordiale du point de vue du droit constitutionnel des libertés. D’abord, le système moniste a été affermi par une telle ouverture vers l'internationalisation des Droits de l'homme. Le rapprochement entre le juge national et la norme internationale semble susceptible d'amorcer les deux processus suivants, mais dans le sens opposé: l'internationalisation des droits et des libertés d'une part, et la nationalisation du droit international des libertés de l'autre. Une telle interaction ne manque pas d'exercer ses effets positifs sur la culture juridique de la magistrature dont la formation est essentiellement calquée sur la notion de loi. Il convient d'ajouter enfin que les destinataires de l'article 90 (dernière phrase) ne sont pas limités aux organes juridictionnels. Toutes les instances ayant la capacité d'appliquer la loi doivent réagir conformément à cette implication constitutionnelle.

\section{B. Les réformes législatives}

Afin de concrétiser la plupart des dispositions constitutionnelles et de les mettre en pratique, une série de lois ont été révisées et certaines renouvelées à partir de 2002. La révision des lois a été effectuée de manière progressive et sous le titre « les ensembles de lois d'harmonisation ». Concernant la liberté d'expression, on peut citer :

- La première loi ${ }^{17}$, destinée à modifier quelques dispositions du Code pénal, du Code de procédure pénale, ainsi que de la loi pour la lutte contre le terrorisme, afin d'élargir la liberté d'expression ;

- La deuxième loi ${ }^{18}$, destinée à la modification de huit lois, telles que la loi sur les associations, la loi sur les réunions et manifestations, la loi sur les partis politiques, la loi de la presse...;

- La troisième loi $^{19}$, qui concerne les droits culturels : l'apprentissage de langues et dialectes utilisés par les citoyens dans leur vie quotidienne et la diffusion d'émissions à la télévision et à la radio dans ces langues et dialectes;

- La quatrième $\operatorname{loi}^{20}$, relative à l'utilisation collective de la liberté d'expression : associations, partis politiques et certains droits collectifs...

Le nouveau Code pénal ${ }^{21}$ a supprimé certaines dispositions susceptibles d'introduire le délit d'opinion. Toutefois, il ne manque pas de dispositions qui constituent une menace sur la liberté d'expression. Plusieurs

16. Comme exemple, il convient de signaler l'affaire Refah Partisi et autres c. Turquie, 13 février 2003, requêtes $n^{\circ}$ 41340/98, 41342/98, 41343/98 et 41344/98, arrêt de la Grande Chambre.

17. Bazı Kanunlarda Değişiklik Yapılmasına Dair Kanun (loi relative à la modification de certaines lois), nº 4744, ta.6.O2. 2002, R.G. : 19.02.2002-24676.

18. Çeşitli Kanunlarda Değişiklik Yapılmasına Ilişkin Kanun (loi relative à la modification de diverses lois), nº 4748, 26.03.2002, R.G. : 9.04.2002-24721.

19. Çeşitli Kanunlarda Değişiklik Yapılmasına Dair Kanun (loi relative à la modification de diverses lois), nº 4771, 3.08.200, R.G. : 9.08.2002-24841.

20. Çeşitli Kanunlarda Değişiklik Yapılmasına IliskinKanun (loi relative à la modification de diverses lois), nº 4778, 02.01.2003, R.G. : 11.01.2003-2499o.

21. Le Code pénal de 1926 a été remplacé par un nouveau Code pénal n ${ }^{\circ} 5237$ du 26 septembre 2004, entré en vigueur le $1^{\text {er }}$ juin 2005 (JO, 12 octobre 2004 , 25611). 
articles du nouveau Code pénal peuvent être signalés, comme les articles 125, 132, 214, 215, 216, 226, 227, 285, 301, $318 \ldots$ et tout spécialement les articles 216 et 301 qui sont à l'origine de plusieurs procès organisés contre des personnes se réclamant de la liberté d'expression.

\section{L'évolution jurisprudentielle et la pratique}

L'évolution jurisprudentielle peut être aussi expliquée par un double effet : l'amélioration du droit positif et les arrêts de la Cour EDH. La référence accrue aux instruments internationaux relatifs aux Droits de l'homme s'y ajoute à partir de 2004, suite à la modification constitutionnelle, qui vise à accorder la primauté aux instruments internationaux par rapport à la loi.

La consolidation constitutionnelle des Droits de l'homme et sa juridictionnalisation au niveau européen ainsi que le processus d'internationalisation peuvent être conçus comme des facteurs cumulatifs sur l'évolution jurisprudentielle concernant la liberté de pensée et d'expression.

Il convient donc de se demander jusqu'à quel point les divergences soulevées entre le juge national et le juge européen ont été éliminées. Autrement dit, est-ce que l'évolution de la jurisprudence nationale a atteint un stade pour lequel il serait possible de parler de convergences plutôt que de divergences?

Il convient donc d'aborder ce thème sous l'optique des restrictions énoncées dans l'article 10 de la CEDH.

\section{A. La pratique de la Cour européenne des Droits de l'homme}

L'article 10 de la CEDH prévoit trois séries de restrictions: d'une part, la sécurité nationale, l'intégrité territoriale ou la sûreté publique, la défense de l'ordre et la prévention du crime; d'autre part, la protection de la santé ou de la morale; et, enfin, la protection de la réputation ou des droits d'autrui pour empêcher la divulgation d'informations confidentielles ou pour garantir l'autorité et l'impartialité du pouvoir judiciaire ${ }^{22}$. Les affaires concernant la Turquie figurent plus particulièrement parmi la première série de restrictions.
D'après la Cour EDH, l'espionnage et le terrorisme $^{23}$, l'incitation au terrorisme ou l'approbation du terrorisme ${ }^{24}$, la subversion ${ }^{25}$, les partis politiques indépendantistes ou séparatistes ${ }^{26}$, l'incitation à la désertion des militaires ou des forces armées ${ }^{27}$, peuvent constituer des menaces contre la sécurité nationale ${ }^{28}$.

La Cour EDH opère une distinction entre la situation « urgente» et celle «normale» de la sécurité nationale. D'après la Cour EDH, les mesures prises au titre de l'article 15 pour la protection de la sécurité nationale nécessaires et proportionnées - ne violaient pas la Convention, dans la mesure où l'État était en droit de protéger l'ordre public face au danger menaçant la vie de la nation ${ }^{29}$

Pour les affaires concernant la Turquie, il convient d'abord de préciser que l'intégrité territoriale de l'État et la menace du terrorisme constituent l'élément central de la notion de la sécurité nationale. Les cours de sûreté d'État (CSE) ont été instaurées par la Constitution de $1982{ }^{30}$ pour juger les infractions considérées comme terroristes et remettant en danger l'intégrité indivisible de l'État, telles que les activités visant à provoquer la sécession d'une partie du territoire national, l'appartenance à une organisation illégale, l'aide ou l'assistance à une telle organisation et le trafic de drogue et d'armes, etc. D'après l'article 9 de la loi relative à la création des $\operatorname{CSE}^{31}$, ces cours sont compétentes pour connaître des infractions à l'article 312 du Code pénal interdisant l'incitation à la haine et à l'hostilité et celles qui sont en rapport avec les événements ayant nécessité la proclamation de l'état d'urgence, dans les régions où l'état d'urgence a été décrété en vertu de la Constitution. Elles jugent également des infractions qui touchent directement la sécurité intérieure ou extérieure de l'État, ou qui sont commises contre la République de Turquie, l'unité indivisible de l'État avec son territoire et sa nation, et contre l'ordre libre et démocratique.

En matière de liberté d'expression en Turquie, la quasi-totalité des condamnations pénales, dont les requérants ont fait l'objet, parce qu’ils avaient exprimé leurs idées et opinions sur la politique menée par le Gouvernement, ont été prononcées par les CSE. Dans l'affaire Saday c. Turquie ${ }^{32}$, la Cour EDH a déclaré que la condamnation immédiate du requérant par la Cour de sûreté de l'État pour avoir exprimé dans sa plaidoirie ses opinions et ses critiques sur la légitimité de cette cour

22. Pour le détail, voir la Revue trimestrielle des Droits de l'homme, numéro spécial La Liberté d'expression, son étendue et ses limites, $\mathrm{n}^{\mathrm{o}}{ }^{13}, \mathrm{1}^{\mathrm{er}}$ janvier 1993 .

23. Cour EDH, Klass et autres c. Allemagne, 6 septembre $1978, \mathrm{n}^{\circ}$ 5029/71, série A-28.

24. Cour EDH (GC), Zana c. Turquie, 29 septembre 2008, $\mathrm{n}^{\circ} 332 / 57$.

25. Cour EDH, Leander c. Suède, 26 mars 1987, $\mathrm{n}^{\circ}$ 9248/81, série A-16.

26. Cour EDH (GC), Parti communiste unifié de Turquie et autres c. Turquie, 30 janvier 1998, $\mathrm{n}^{\circ}$ 19392/92. Cour EDH (GC), Parti socialiste et autres c. Turquie, 25 mai $1998, \mathrm{n}^{\circ} 21237 / 93$.

27. Cour EDH (GC), Grigoriades c. Grèce, 25 novembre 1997, nº 24348/94.

28. Pour le détail, voir Ü. Kılınç, La Liberté d'expression en Turquie à l'épreuve de la Convention européenne des Droits de l'homme, Thèse de doctorat, Université de Strasbourg, 18 avril 2009, p. 327-339.

29. Cour EDH, Lawless c. Irlande ( $\left.\mathrm{n}^{\circ} 3\right), 1^{\text {er }}$ juillet $1961, \mathrm{n}^{\circ} 332 / 57$, série A, p. 43-63.

30. Elles ont été supprimées par la modification constitutionnelle en 2004.

31. La loi $\mathrm{n}^{\circ} 2845$ du 16 juin 1983 relative à la création des CSE et à la réglementation de la procédure devant elles.

32. Cour EDH, Saday c. Turquie, 30 mars 2006, nº 32458/96. 
lors de l'audience n'était pas conforme à l'article 10 de la Convention $^{33}$.

Par ailleurs, le Conseil de l'Europe a adopté la Convention pour la prévention du terrorisme, qui a été ouverte à la signature des États membres le 16 mai $2005^{34}$.

La Cour EDH constate que le terrorisme se développe en Europe au cours des dernières années et que les «sociétés démocratiques se trouvent menacées de nos jours $[. .$.$] par le terrorisme { }^{35}$. Sans doute, affirme-telle que nul ne peut justifier un acte terroriste en invoquant les Droits de l'homme ${ }^{36}$. Cependant, d'après la Cour EDH, comme chaque individu, les terroristes ou ceux qui sont soupçonnés de terrorisme jouissent des droits et libertés consacrés par la Convention puisque l'article 10 qui garantit la liberté d'expression à «toute personne» ne fait pas cas du but recherché, ni du rôle que les personnes physiques ou morales ont joué dans l'exercice de cette liberté ${ }^{37}$. Ils peuvent sans doute s'en prévaloir lorsqu'ils ont été maltraités ou torturés, arrêtés, détenus, condamnés ou privés de leurs droits à la vie, à la vie privée et familiale, et à la liberté d'expression en violation des dispositions conventionnelles ${ }^{38}$.

Lorsqu'il s'agit d'appel à la haine ou à la violence, il n'est pas nécessaire de rechercher s'il y a un lien avec le terrorisme, étant donné que la violence et la haine ne sont pas admissibles dans une démocratie. D’après la Cour EDH, les responsables d'un parti politique qui incitent à la violence, ou proposent un projet politique qui ne respecte pas une ou plusieurs règles de la démocratie ou vise la destruction de celle-ci ou méconnaît des droits et libertés consacrés dans la Convention, ne peuvent bénéficier de la protection de la Convention ${ }^{39}$.

La question devient plus complexe lorsqu'il y a des liens entre l'expression des idées politiques et une organisation qualifiée de terroriste. La Cour EDH, dans les affaires relatives à la liberté d'expression en Turquie, applique le critère de l'incitation à la violence lorsque les dirigeants d'une organisation considérée comme terroriste expriment leur point de vue sur les problèmes politiques dans le pays ${ }^{40}$. Pour déterminer si les propos dans leur ensemble peuvent être considérés comme une incitation à la violence, la Cour fait attention aux termes employés, au contexte dans lequel leur publication s'ins$\mathrm{crit}^{41}$, et à la place qu'occupe la personne qui prononce les propos ${ }^{42}$. D'après la Cour européenne, le fait de publier dans un journal les entretiens réalisés avec le dirigeant d'une organisation armée ne saurait en soi justifier l'ingérence dans le droit des requérants à la liberté d'expression ${ }^{43}$. Compte tenu de l'absence de violence, la Cour a affirmé que le public a le droit d'être informé d'une autre manière sur la situation dans le Sud-Est de la Turquie pour comprendre la psychologie des personnes constituant les forces vives de l'opposition à la politique officielle appliquée dans la région et d'apprécier les enjeux du conflit. Selon la Cour EDH, la condamnation du requérant pouvait dissuader la presse de contribuer à un débat ouvert sur des questions d'intérêt public ${ }^{44}$.

Toutefois, dans les affaires ultérieures, la Cour européenne a constaté que « la liberté d'expression ne permet pas à la presse de servir de tribune de transmission des idées de violence à travers, entre autres, les déclarations des membres des organisations interdites ${ }^{45}$. Elle a aussi souligné que «le droit de communiquer des informations ne (peut) servir d'alibi ou de prétexte à la diffusion de déclarations de groupements terroristes ${ }^{46}$. D'après la Cour européenne, même si les journalistes ne sont pas personnellement associés aux déclarations exprimées par les dirigeants de l'organisation, ils partagent "indirectement les "devoirs et responsabilités" que les auteurs assument lors de la diffusion de leurs opinions auprès du public ${ }^{47}$.

En effet, la Cour européenne prend plus souvent en compte plus particulièrement la personnalité des auteurs des propos que leur contenu et le contexte dans lequel ils ont été prononcés ainsi que le moyen utilisé ${ }^{48}$. Par contre, d'après une approche nuancée, «la Cour porte une attention particulière aux termes employés dans l'article en question. [...] Pour la Cour, l'emploi d'une telle expression révèle une nette intention de légitimer le recours à la violence. À cet égard, elle rappelle que l'idéologie du PKK, telle que présentée par Abdullah Öcalan [le chef du PKK], implique le recours à la violence et, la lutte et la résistance armée sont présentées par celui-ci comme une "défense légitime" " ${ }^{49}$.

\footnotetext{
33. Pour le détail sur la pratique des CSE, voir Ü. Kılınç, La Liberté d'expression..., p. 326 sq.

34. Cette Convention a été signée par la Turquie le 19 janvier 2006, mais non encore ratifiée.

35. Cour EDH, arrêt Class et autres c. Allemagne, 6 septembre 1978, $\mathrm{n}^{\circ}$ 5029/71, $\$ 48$.

36. Lignes directrices du Comité des ministres du Conseil de l'Europe sur les Droits de l'homme et la lutte contre le terrorisme, $804^{\mathrm{e}}$ réunion, 11 juillet 2002.

37. Pour les arrêts de la Cour EDH, voir Ü. Kılınç, La Liberté d'expression..., p. 348-350.

38. Citons quelques arrêts de la CEDH comme: Tomasi c. France, 27 août 1992, $\mathrm{n}^{\circ}$ 12850/87, \$ 115; Fox, Campbell et Hartley c. Royaume-Uni, 30 août 1990, $\mathrm{n}^{\circ} 12244 / 86, \mathrm{n}^{\circ} 12245 / 86$ et $\mathrm{n}^{\circ}$ 12383/86, $\$ 34 ;$ Baştımar et autres $c$. Turquie, 5 décembre 2006, $\mathrm{n}^{\circ}$ 74337/01; Tan c. Turquie, 3 juillet $2007, \mathrm{n}^{\circ} 9460 / 03$.

39. Cour EDH (GC), Refah Partisi (Parti de la Prospérité) et autres c. Turquie, 13 février 2003, $\mathrm{n}^{\circ} 41340 / 98,41342 / 98$ et $41344 / 98, \$ 98$.

40. Voir Ü. Kılınç, La Liberté d'expression..., p. 356-357.

41. Cour EDH, Özgür Gündem c. Turquie, 16 mars 2000, $\$ 63$.

42. Cour EDH (GC), Zana c. Turquie, 25 novembre 1997.

43. Cour EDH (GC), Sürek et Özdemir c. Turquie, 8 juillet 1999.

44. Cour EDH (GC), Sürek c. Turquie ( ${ }^{\circ}{ }^{2}$ ), 8 juillet 1999, $\$ 39-42$.

45. Cour EDH, déc. Demirel c. Turquie, 3 mai 2007, $\mathrm{n}^{\circ}$ 12166/03; Karakoyun et Turan c. Turquie, 11 décembre 2007, $\mathrm{n}^{\circ} 18482 / 03, \$ 30$.

46. Cour EDH, Falakoğlu et Saygılıc. Turquie, 23 janvier 2007, nº 22147/02 et 24972/03, \$34.

47. Cour EDH, Erdal Taş $\left(\mathrm{n}^{\circ} 3\right), 20$ septembre 2007, $\mathrm{n}^{\circ}$ 17445/02, $\$ 23$; Falakoğlu et Saygılı c. Turquie, $\$ 34$.

48. Cour EDH, Erdal Taş $\left(\mathrm{n}^{\circ} 3\right), 20$ septembre 2007, $\mathrm{n}^{\mathrm{o}} 17443 / 02, \$ 21-22$.

49. Cour EDH, Erdal Taşc. Turquie (n $\left.{ }^{\circ} 2\right), 20$ septembre 2007, $\mathrm{n}^{\circ}$ 13021/02, $\$ 20-21$.
} 
Toutefois, dans ses arrêts plus récents, la Cour EDH reflète une tendance vers un élargissement de la liberté d'expression, en affirmant que le discours ou l'article incriminé «n'était pas susceptible de favoriser la violence en insufflant une haine profonde et irrationnelle envers des personnes identifiées ${ }^{50}$.

Les actions telles qu'une grève de la faim et la distribution de tracts n'étant pas considérées comme un appel à la violence bénéficient aussi, d'après l'appréciation de la Cour EDH, de la liberté d'expression ${ }^{51}$ : « Le requérant allègue que sa condamnation au pénal pour avoir distribué un tract et avoir pris la décision de mener une grève de la faim a porté atteinte à ses droits à la liberté d'expression et d'association. " ${ }^{52}$ D' après la Cour, «à l'époque des faits de la cause, des grèves de la faim étaient menées par les détenus des prisons pour protester contre les conditions de détention imposées selon le nouveau régime carcéral de type $\mathrm{F}$, prévoyant des unités de vie d'une à trois personnes au lieu de dortoirs [...] le requérant et les membres de l'association, en utilisant ces deux types d'action, ne faisaient que sensibiliser l'opinion publique au sujet des grèves de la faim menées dans les prisons et les conditions de détention, selon leurs dires, déplorables des prisons de type $\mathrm{F}[\ldots]{ }^{53}$.

Parmi les derniers arrêts de la Cour EDH, il convient de citer l'affaire Görkan afin de souligner la sensibilité de la Cour vis-à-vis de la liberté de presse ${ }^{54}$ : "Selon le requérant, les policiers vérifièrent, par une communication avec le centre de police (merkez), qu'il n'était pas une personne recherchée, et que le numéro du journal qu'il était en train de vendre n'avait pas fait l'objet d'une décision de saisie $[. ..]{ }^{55}$ Quant à la Cour, elle estime que le contrôle policier effectué en l'espèce, accompagné d'une « invitation au poste de police» qui peut être assimilée à une restriction de liberté de par l'élément coercitif qu'elle comporte, dépourvue de toute raison plausible ou motif raisonnable, constitue bien « une ingérence dans la liberté de communiquer des informations du requérant ${ }^{56}$.

\section{B. L'évolution de la jurisprudence nationale}

Les dynamiques de l'évolution jurisprudentielle sont principalement les arrêts de la Cour EDH et les lois d'harmonisation ainsi que l'article 90 de la Constitution. On assiste tout d'abord à l'application subsidiaire de l'article 10 de la Convention par rapport à la législation nationale. Par ailleurs, il s'agit d'une application avec effet de primauté de l'article 10 de la Convention par rapport à la législation nationale et à la multiplication des citations des arrêts de la Cour EDH.

\section{La CEDH comme norme secondaire}

La Cour constitutionnelle d'Ankara se référait depuis les années 1960 à la CEDH dans ses arrêts dans le cadre de son contrôle de constitutionnalité des lois. Toutefois, c'était la Constitution qui était utilisée comme norme de référence, alors que la $\mathrm{CEDH}$ et la jurisprudence des organes de Strasbourg constituaient plutôt des «sources d'inspiration» dans la jurisprudence de la Cour constitutionnelle. À partir des années 1990, les arrêts relatifs à la dissolution des partis politiques ont donné l'occasion à la Cour constitutionnelle de s'approprier davantage les dispositions de la CEDH. La notion de Droits de l'homme qui figure parmi les dispositions non modifiables de la Constitution en tant qu' «État respectueux des Droits de l'homme» fut également, pour la Cour constitutionnelle, le fondement juridique de la référence à la Convention et à la jurisprudence de la Cour européenne. Pour la Cour constitutionnelle, un État ne respectant pas les obligations découlant du droit international ne peut pas être considéré comme un «État respectueux des Droits de l'homme », ni un «État de droit » au sens de l'article 2 de la Constitution ${ }^{57}$.

La Cour de cassation eut une approche plus réticente vis-à-vis de la CEDH par rapport à celle de la Cour constitutionnelle. Dans l'application de certaines dispositions du Code pénal et de la loi sur la lutte contre le terrorisme, susceptibles de créer le délit d'opinion, la Cour de cassation a donné la priorité à des notions telles que l'État unitaire $^{58}$ et l'État laïc ${ }^{59}$ face à la liberté d'expression.

Quant à la jurisprudence du Conseil d'État, une approche plus ouverte à la CEDH par rapport à la Cour constitutionnelle et à la Cour de cassation doit être notée.

\section{L'application de l'article 10 de la CEDH}

Les articles 15 et 16 de la Constitution concernant les Droits de l'homme renvoient au droit international. D'après l'article 15, les restrictions et la suspension des Droits de l'homme en cas d'état d'exception ne doivent pas enfreindre les obligations découlant du droit international. La Cour constitutionnelle, amenée à se prononcer sur l'expression «ne pas violer les obligations découlant du droit international », a précisé que le législateur était

50. Cour EDH, Kanat et Bozan c. Turquie, 21 octobre 2008, $\mathrm{n}^{\circ}$ 13799/04, \$19; Ísak Tepe c. Turquie, 21 octobre 2008, $\mathrm{n}^{\circ} 17129 / 02, \$ 24$.

51. Cour EDH, Kara c. Turquie (requête ${ }^{\circ}$ 22766/04), 30 juin 2009 (définitif : 30 septembre 2009).

52. Arrêt Kara, $\$ 18$.

53. Arrêt Kara, $\$ 36$

54. Cour EDH, Görkan c. Turquie (requête $\mathrm{n}^{\circ}$ 13002/05), 16 mars 2010.

55. Arrêt Görkan, $\$ 6$.

56. Arrêt Görkan, \$37.

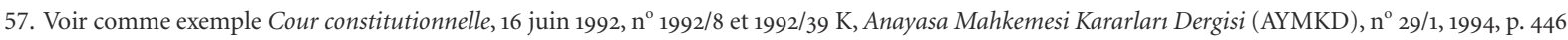

58. Voir comme exemple Ass. Plén. (crim.), 25 mai 2005, nº 2005/9-320 E et 2005/355 K, Yargitay Kararlar Dergisi (YKD), Ekim 2005, Cilt 31, $\mathrm{n}^{\circ}$ 10, p. 15011525

59. Cass. crim., $8^{\mathrm{e}}$, 23 septembre 1998, nº 1998/10296 E et 1998/11672 K, YKD, Kasım 1998, Cilt 24, nº 11, p. 1728. 
tenu de respecter, en cas d'état d'exception, les principes généraux du droit international ${ }^{60}$.

L'article 16 de la Constitution prévoit aussi que les restrictions apportées aux droits et libertés des étrangers doivent être conformes au droit international ${ }^{61}$.

Le Conseil d'État, sous le régime constitutionnel précédent, avait déjà censuré un acte gouvernemental au nom de la liberté d'expression ${ }^{62}$. En se référant à l'acte final d'Helsinki du $1^{\text {er }}$ août 1975, il a annulé un arrêté d'expulsion pris et adopté contre les journalistes d'une télévision suédoise. En ce qui concerne l'application de la $\mathrm{CEDH}$ en matière de liberté d'expression, la priorité est accordée aux articles de la Convention par rapport au droit national dans la jurisprudence du Conseil d'État ${ }^{63}$.

La pratique prioritaire de l'article 10 de la CEDH n'est pas exceptionnelle dans la jurisprudence du Conseil d’État: «[...] En l'espèce, les idées exprimées dans la carte postale, sur laquelle est fondé le licenciement du demandeur [...], sont ses souhaits et désirs sur différents sujets. Il est admis que le fait que toute personne puisse exprimer ses idées, sans incitation ouverte et concrète ou encouragement à commettre un délit, est un principe primordial et fondamental de la société démocratique. ${ }^{64}$

D’après le juge administratif, il était manifeste que les idées et opinions exprimées dans la carte postale n'avaient pas dépassé les limites au sens du second paragraphe de l'article 10 de la Convention et que le demandeur avait exercé le droit à la liberté d'expression que les Conventions internationales lui reconnaissaient.

Un troisième exemple peut être donné : l'Assemblée plénière du Conseil d'État a annulé l'acte ministériel du 23 octobre 2007 qui visait à interdire les émissions et diffusions de Yașam TV. Elle a estimé qu'il était nécessaire d'examiner l'affaire à la lumière de l'article 10 de la Convention et des dispositions de la Constitution concernant la liberté d'expression. L'Assemblée plénière, après avoir énoncé les critères adoptés par la Cour de Strasbourg, a souligné que l'acte administratif en question ne répondait pas aux exigences de clarté inhérentes à la notion de «loi» au sens de l'article 10, puisqu'il ne précisait pas quel type d'émission ou de diffusion pouvait faire l'objet de l'interdiction. Dès lors, le pouvoir qu'exerçait le ministère pour suspendre ou interdire des émissions de radio et télévision par le biais du décret en question méconnaissait la liberté de la presse ${ }^{65}$.
En ce qui concerne les arrêts de la Cour de cassation, il convient de rappeler tout d'abord que la plupart des arrêts de la Cour EDH relatifs à la liberté d'expression se prononcent sur les arrêts des juridictions judiciaires. Pour cette raison, la Cour de cassation joue un rôle déterminant dans la mise en œuvre de la Convention et de ses règles. On assiste à une évolution permanente dans la jurisprudence de la Cour de cassation relative à la pratique de l'article 10 de la Convention. Elle prend également en compte de plus en plus les arrêts de la Cour EDH et ce d'autant plus à la suite de l'ajout d'une phrase à l'article 90 de la Constitution accordant une supériorité aux instruments internationaux relatifs aux Droits de l'homme par rapport à la loi.

La Cour de cassation a d'abord reconnu la primauté des décisions de la Commission européenne des Droits de l'homme par rapport à ses arrêts ${ }^{66}$ et, pour protéger la liberté d'expression dans l'optique des arrêts de la Cour $\mathrm{EDH}$, elle a ensuite abandonné sa jurisprudence antérieure $^{67}$. Enfin, la Cour de cassation a fondé sa décision sur l'article 10 de la Convention, considérant que les propos de l'accusé constituaient une critique forte à l'égard du président de la République et devaient entrer dans le cadre de l'exercice de la liberté d'expression ${ }^{68}$.

L'incitation à la haine et à l'hostilité et l'incitation à la violence peuvent être perçues comme les deux éléments principaux susceptibles de concrétiser l'évolution de la jurisprudence de la Cour de cassation en ce qui concerne la liberté d'expression. L'article du Code pénal qui interdisait l'incitation à la haine et à l'hostilité était l'une des sources principales de la condamnation de la Turquie sur la base de l'article 10 de la CEDH du fait qu'il a été l'objet d'une application stricte de la part de la Cour de cassation ${ }^{69}$.

La Cour de cassation a appliqué les critères qui découlent de la jurisprudence de la Cour EDH après avoir estimé que les dispositions de la Constitution et celles de la Convention devaient être examinées ensemble $^{70}$. D'après la Haute Cour, l'ingérence dans le droit à la liberté d'expression du requérant qui avait exprimé de vives critiques à l'égard du principe de laïcité n'était pas nécessaire dans une société démocratique au sens du second paragraphe de l'article 10 de la Convention européenne $^{71}$. La Cour de cassation a infirmé le jugement de première instance au motif que celle-ci avait condamné

60. Cour constitutionnelle, 10 janvier 1991, n 1990/25 E et 1991/1 K, AYMKD, nº 27/1, 1993, p. 96.

61. La Cour constitutionnelle, dans son arrêt du 13 juin 1985, a confirmé que si les textes internationaux concèdent à tous les États le droit de restreindre les droits et libertés fondamentaux des étrangers, les restrictions doivent être compatibles avec le droit international, comme l'exige l'article 16 (arrêt du 13 juin 1985, $n^{\circ}$ 1984/14 et 1985/7 K, AYMKD, n 21, 1986).

62. Conseil d'État, $12^{\mathrm{e}}{ }^{\mathrm{C}}$ Chambre, le 24 avril 1978, ${ }^{\circ}$ 1977/1349 et 1978/955 K, Danıştay Dergisi, n ${ }^{\circ}$ 30-31, 1978, \$ 50-53.

63. Le Conseil d'État se réferre également aux autres dispositions de la Convention.

64. Conseil d'État, $5^{\mathrm{e}}$ Chambre, le 22 mai 1991, nº 1986/1723 E et 1991/933 K, Danıştay Dergisi, nº 84-85, 1992, p. 326.

65. Pour le détail de la décision du $1^{\text {er }}$ novembre 2007, voir Ü. Kılınç, La Liberté d’expression ..., p. 386-387.

66. Cour de cassation, Ass. Plén. (crim.), le 17 mars 1998, nº 1998/6-18 E et 1998/91 K et 1998/91 K (non publié).

67. Cour de cassation, crim. $9^{\mathrm{e}}$ Chambre, le 4 novembre 2003, $\mathrm{n}^{\circ}$ 2003/1704 E et 2003/1971 K (non publié).

68. Cour de cassation, crim. $9^{\mathrm{e}}$ Chambre, le 24 mars 2004, n ${ }^{\circ}$ 2004/3542 E et 2004/4545 K (non publié).

69. Pour le détail, voir Ü. Kılınç, La Liberté d'expression..., p. 473-478.

70. Cour de cassation, Ass. Plén. (crim.), 23 novembre 2004, nº 2004/8-130 E et 2004/206 K (Yargitay Kararlar Dergisi, Mart-2005, Cilt 31, $n^{\circ} 7$ ).

71. «[...] car il s'agit d'un des rares arrêts rendus par la haute juridiction judiciaire qui ait développé de manière détaillée l'ensemble des faits, de la procédure et des motifs basés sur la jurisprudence de la Cour de Strasbourg. » Ü. K1lınç, La Liberté d'expression..., p. 390. 
l'accusé pour propagande séparatiste sans tenir compte du fait qu'il n'avait pas appelé à l'usage de la violence ${ }^{72}$.

Toutefois, la jurisprudence de la Cour de cassation manque de stabilité du point de vue de l'application de l'article 10 et du critère de l'incitation à la violence. La Cour de cassation semble renoncer à rechercher, dans un arrêt suivant, si l'accusé avait incité, dans ses déclarations, à la haine et à la violence. La Cour de cassation, qui s'est prononcée dans le cadre de l'article 312 du Code pénal, a affirmé que, même si les propos utilisés par l'accusé dans son article de journal n'appelaient pas à la haine et à la violence, ils diffamaient et portaient atteinte au principe de la laïcité qui ne saurait être remis en cause, même sous prétexte de l'exercice de la liberté d'expression ${ }^{73}$. Dans un autre arrêt, relatif à l'identité turque, infraction prévue par l'article 159 du Code pénal, la Cour de cassation n’a pas non plus appliqué le critère de l'incitation à la violence ${ }^{74}$. Ces deux arrêts auraient marqué « une régression de la jurisprudence de la Cour de cassation en matière de liberté d'expression [... et seraient] contraire[s] à la jurisprudence de la Cour de Strasbourg en matière de liberté d'expression ${ }^{75}$.

\section{Le nouveau Code pénal et l'application du critère de danger clair et proche}

Concernant la jurisprudence de la Cour de cassation développée à la suite du renouvellement du Code pénal $^{76}$, il convient d'abord de signaler que les deux articles du Code cités ci-dessus susceptibles d'introduire le délit d'opinion ont été rédigés de nouveau tout en maintenant l'esprit de ces dispositions. Le nouveau Code pénal a eu une incidence positive sur un certain nombre de domaines liés aux Droits de l'homme, en particulier la liberté d'expression ${ }^{77}$. Quant au contenu du nouveau Code pénal, il a limité, dans une certaine mesure, le champ d'application de certains des articles qui ont été utilisés pour condamner ceux qui exprimaient des opinions non violentes. Le nouvel article 216, qui correspond largement à l'article 312 de l'ancien, prévoit que les individus ne peuvent être condamnés en vertu de cet article que si leur «incitation à l'hostilité et à la haine» constitue un «danger clair et proche». La peine minimale pour l'infraction de diffamation a été réduite dans le nouveau code ${ }^{78}$.

Cependant, l'article 301 est quasiment calqué sur l'article 159 de l'ancien Code pénal ${ }^{79}$. Pour cette raison, sa pratique a souvent abouti à créer le délit d'opinion. En fait, la notion de "turquicité» (türklük), à défaut d'être définie juridiquement de manière claire et concrète, fut la base des poursuites et des condamnations liées à l'expression des opinions non violentes. Il sanctionnait tout outrage proféré à l'encontre de la «turquicité », de la République et des organes et institutions de l'État. L'article 301 ne cesse d'être utilisé pour poursuivre ceux qui font usage du droit à la liberté d'expression des opinions non violentes alors qu'il comprenait une disposition stipulant que l'expression d'opinions critiques ne constitue pas un délit ${ }^{80}$.

L'article 301, très contesté par les défenseurs des Droits de l'homme, a été amendé afin de limiter la marge d'appréciation des juges concernant l'application et l'interprétation de cette disposition. Ainsi, la notion de « turquicité » a été remplacée par la « nation turque » ${ }^{81}$, et les autorités judiciaires ne peuvent engager les poursuites pénales en vertu de l'article 301 qu'après l'autorisation du ministre de la Justice comme cela était prévu dans l'article 159 du Code pénal précédent ${ }^{82}$. Malgré cela, l'article 301 est encore susceptible d'être utilisé de manière à entraver la liberté d'expression ${ }^{83}$.

Des journalistes, universitaires et romanciers, ainsi que des défenseurs des Droits de l'homme, ont été récemment poursuivis pour avoir violé les articles 216 et $301^{84}$.

72. Cour de cassation, crim. $9^{\mathrm{e}}$, le 22 juin $2005, \mathrm{n}^{\mathrm{o}} 2004 / 9121 \mathrm{E}$ et $2005 / 4215 \mathrm{~K}$.

73. Cour de cassation, Ass. Plén. (crim.), 15 mars 2005, nº 2004/8-201 E et 2005/30 K (Yargitay Kararlar Dergisi, Temmuz 2005, Cilt 31, $n^{\circ}$ 7). C’est l'un des arrêts de la Cour de cassation qui contiennent une explication très détaillée en ce qui concerne la référence à la jurisprudence de la Cour EDH.

74. Cour de cassation, Ass. Plén. (crim.), 11 juillet 2006, nº 2006/9-169 E et 2006/184 K.

75. Ü. Kilınç, La Liberté d'expression..., p. 392.

76. La loi $\mathrm{n}^{\mathrm{o}} 5237$ du 26 septembre 2004 (JO, 12 octobre 2004, $\mathrm{n}^{\mathrm{o}} 25611$ ) est entrée en vigueur le $1^{\text {er }}$ juin 2005.

77. «Plusieurs personnes purgeant une peine d'emprisonnement suite à une condamnation fondée sur les articles 159 et 312 de l'ancien Code pénal ont été remises en liberté. » Ü. Kilınç, La Liberté d'expression..., p. 486.

78. D'après l'article 216, est « passible d'une peine d'un an à trois ans d'emprisonnement quiconque incite publiquement à la haine et à l'hostilité une partie de la population appartenant à des classes sociales, races, religions, sectes ou régions contre une autre partie, de manière à créer un danger clair et présent pour la sécurité publique $[\ldots] »$.

79. L'article 301 du nouveau Code pénal énonce : « est passible d'une peine de six mois à trois ans d'emprisonnement quiconque offense publiquement la turquicité, la République ou la Grande Assemblée nationale de Turquie; est passible d'une peine de six mois à deux ans d'emprisonnement quiconque offense publiquement le Gouvernement de la République de Turquie, les organes judiciaires, les forces militaires, ou bien la sûreté de l'État; la peine sera augmentée d'un tiers lorsque la nation turque a été offensée à l'étranger par un citoyen turc; l'expression d'opinions visant à critiquer ne constitue pas un délit $[\ldots] »$.

80. Pour la pratique de l'article, voir Ü. Kılınç, La Liberté d'expression..., p. 486-491.

81. L'article 1 de la loi n 5759 du 30 avril 2008 relative à la modification du Code pénal turc (JO, 8 mai 2008, $\left.\mathrm{n}^{\circ} 26870\right)$.

82. Selon la déclaration de M. le ministre de la Justice, le nombre de dossiers pour lesquels les autorités judiciaires ont demandé l'autorisation est de 1252 en date du 30 avril 2010. Parmi ces dossiers, le nombre de dossiers autorisés n’est que de 83. Voir Antenna, Haftalık Düşünce Özgürlüğü Bülteni (Bulletin hebdomadaire pour la liberté de pensée), nº 22/10, 28 mai 2010.

83. Pour le détail, voir Düşünceye Özgürlük 2009 (Liberté de pensée 2009), Düşünce Suçuna karşı Girişim (Initiative contre le délit d’opinion), Istanbul, 2009. Cette initiative publie, chaque année depuis 1995, au moins un livre afin de faire le bilan des délits d'opinion. Elle effectue également une publication hebdomadaire sur le site Internet afin de diffuser des informations sur l'actualité des poursuites et des procès engagés contre les intellectuels et journalistes.

84. Pour les procès engagés contre plusieurs intellectuels, de M. Hrant Dink à Orhan Pamuk, voir Ü. Kılınç, La Liberté d'expression..., p. 479-491. 
À ce propos, un procès intenté contre deux membres du Conseil consultatif des Droits de l'homme, $(\mathrm{CCDH})^{85}$ accusés d'avoir rédigé et fait adopter un rapport sur les droits des minorités et minorités culturelles, semble tout à fait significatif ${ }^{86}$.

Le procureur d'Ankara a intenté un procès pénal contre deux membres du $\mathrm{CCDH}^{87}$, accusés d'avoir violé les articles 159 et 312 du Code pénal ${ }^{88}$ en dénigrant l'identité turque par la mise en cause de la définition de la citoyenneté et la proposition d'une supra-identité à base territoriale. Ces propos auraient constitué une incitation du peuple à la haine et à l'hostilité (art. 216/1) et une humiliation pour le pouvoir juridictionnel (art.301/2) en raison des critiques formulées à l'encontre des arrêts de la Cour constitutionnelle.

Le juge du tribunal correctionnel d'Ankara a, dès la première audience du 15 février 2006, écarté le chef d'inculpation pour « humiliation du pouvoir juridictionnel » en demandant l'autorisation du ministère de la Justice. À la fin de la troisième audience du 10 mai 2010, le juge d'Ankara a prononcé l'acquittement fondé sur l'incrimination d'incitation à la haine et à l'hostilité $\left(\right.$ art. 216) ${ }^{89}$.

La $8^{\text {e }}$ Chambre criminelle de la Cour de cassation a cassé sur le fond l'arrêt d'acquittement le 12 juillet 2007, sous l'optique du pourvoi en cassation introduit par le procureur principal d'Ankara. Par cet arrêt, rendu public le 12 septembre 2007 , la $8^{\mathrm{e}}$ Chambre criminelle a créé un délit d'opinion typique. Fort heureusement, l'Assemblée plénière criminelle de la Cour de cassation, en annulant l'arrêt rendu par la $8^{\text {e }}$ Chambre, a approuvé le 29 avril 2008 d'une façon définitive l'arrêt d'acquittement concernant l'article $216^{90}$.

Malgré une telle issue positive, «la poursuite pénale contre les deux représentants du $\mathrm{CCDH}$ n'a pas seulement constitué une ingérence dans la liberté d'opinion, mais elle a également porté atteinte à la liberté scientifique et universitaire, car sont poursuivis deux professeurs qui avaient été désignés en leur qualité de spécialistes. Plus grave encore: les rapports du CCDH ont été élaborés dans le cadre d'une fonction déterminée par la loi. Dans ce procès, en effet, une instance des Droits de l'homme (le $\mathrm{CCDH}$ ) s'est trouvée être jugée par une autre instance protectrice des droits de l'Homme (le tribunal/la justice) ${ }^{91}$.

Cependant, le procès en cause a permis à l'Assemblée plénière des chambres criminelles de la Cour de cassation d'interpréter pour la première fois l'article $216 \mathrm{du}$ nouveau Code pénal qui introduit le critère de «danger public clair et imminent", renvoyant à la commission d'un délit fondé sur l'incitation du peuple à la haine et à l'hostilité. D’après l'Assemblée plénière, «Le rapport mentionné et le communiqué de presse ne donnent lieu à aucun risque concret [...] il n'y a eu aucune indignation ou intempérance entre les segments de la société, ni aucun incident spécifique touchant à l'ordre public n'était réalisé ${ }^{92}$.

Le rapport et le communiqué de presse devraient être, selon la Cour, appréciés dans le cadre de la liberté d'expression : «Même s'il est possible d'adopter ou rejeter les propositions du Rapport, ce dernier qui a été élaboré d'une manière scientifique et en vertu d'une loi, ne comprend aucun appel à la violence, dès lors qu'il ne contient aucun danger clair et imminent du point de vue de la sécurité publique ${ }^{93}$.»

Une telle interprétation du critère de « danger public clair et imminent » peut être conçue dans le contexte du droit comparé des libertés : «clear and present danger ${ }^{94}$.

Ceci dit, l'évolution récente dans la jurisprudence en droit interne est marquée par l'application de l'article 90 de la Constitution qui confère la primauté aux traités internationaux par rapport au droit national. Parmi les traités internationaux, CEDH tient une place à part. L'application d'office de la Convention est mise en œuvre par le juge national surtout depuis l'ajout fait à l'article 90 en 2004. Le juge national fonde ses arrêts sur la

85. Le CCDH avait été mis en place par une loi de 2001 en tant qu'institution nationale pour la protection et la promotion des Droits de l'homme sous l'optique des «Principes de Paris », adoptés lors des premières rencontres internationales des Institutions nationales des Droits de l'homme à Paris en 1991, ces principes ont été officiellement consacrés par l'Assemblée générale des Nations Unies en 1993.

86. Pour le détail, voir I. Ö. Kaboğlu, «Le Conseil consultatif des Droits de l'homme devant le tribunal pénal (cas de la Turquie) », Revue de science criminelle et de droit pénal comparé, juillet-septembre 2006, p. 521-535; "Quelques remarques préliminaires à propos d'une institution nationale des Droits de l'homme (le cas de la Turquie) ", Revue trimestrielle des Droits de l'homme, nº 68, 2006, p. 1057-1066.

87. Les professeurs Ibrahim Ö. Kaboğlu (président du Conseil) et Baskın Oran (président du Groupe de travail pour les droits des minorités et minorités culturelles).

88. Respectivement : articles 301/2 et 216/1 du nouveau Code pénal.

89. «La Convention européenne des Droits de l'homme a été au centre des débats... La primauté de la Convention consacrée par la Constitution turque, l'inconstitutionnalité et l'inconventionnalité des dispositions concernées du Code pénal ont été fortement mises en avant. [...] 》 (Compte rendu de M. le Professeur Christian Grellois qui a assisté à l'audience en tant que membre du Groupe d'initiative).

90. Toutefois, la partie du dossier relative à l'article 301 a été renvoyée au tribunal correctionnel d'Ankara afin de la soumettre au ministre de la Justice pour savoir si celui-ci autorise ou non le jugement. Finalement, le ministère de la Justice n'a pas autorisé le jugement. Quant au juge correctionnel, celui-ci a rejeté la demande de l'exception d’inconstitutionnalité de l'article 301/2. Voir I. Ö. Kaboğlu, «L’article 301 : on n’a pu ni se faire juger, ni le faire juger» (traduction de l'article publié dans BirGün: «301: ne yargılanabildik, ne de yargılatabildik», 9 avril 2009).

91. I. Ö. Kaboğlu, «Le Conseil consultatif des Droits de l’homme devant le tribunal pénal», p. 534.

92. Un résumé de l'arrêt de l'Assemblée plénière a été publié en français en annexe de l’article suivant: I. Ö. Kaboğlu, «Le Conseil consultatif des Droits de l'homme devant le tribunal pénal (cas de la Turquie) », Festschrift für zum 75. Geburtstag Ali Ülkü Azrak 75. Yaş Armağanı, Istanbul, 2008, p. 147-150 [inédit] (cet article est une version révisée et mise à jour de l'article publié sous le même titre dans la Revue de science criminelle et de droit pénal comparé).

93. I. Ö. Kaboğlu, «Le Conseil des Droits de l'homme... », p. 149. Pour une analyse de cet arrêt du point de vue du droit pénal, voir T. Y. Sancar, «Ceza Hukukunda “Özgürlükçü” ve "Baskıcı” yaklaşımlar- Yargıtay Ceza Genel Kurulunun “Kaboğlu-Oran” Kararı», Prof. Dr Yılmaz Aliefendioğlu’na Armağan, Ankara, Yetkin yay., 2009, p. 401-433.

94. Voir W. Sadurski, Freedom of Speech and Its Limits, Boston - Londres - Dordrecht, Kluwer Academic Publishers, 2001, p. $203-205$. 
Convention, en l'interprétant à la lumière de la jurisprudence européenne. Il veille donc, de plus en plus, à une application de la Convention en harmonie avec le juge européen et commence à la viser d'office dans les contentieux internes. «Cela permet au juge turc de converger avec le juge européen et d'éviter la condamnation de la Turquie à Strasbourg. » ${ }^{95}$

Au niveau national, il convient de remarquer que la dernière phrase de l'article 90 est une disposition constitutionnelle fortement "socialisée», du fait qu'elle est souvent évoquée non seulement devant les instances juridictionnelles mais à la fois auprès des autorités administratives.

Progrès ou régression en ce qui concerne la liberté d'expression en Turquie? Cette dernière décennie peut être résumée à la fois par ces deux mots: progrès et régression.

Les avancées peuvent être constatées sur le plan du droit positif, dans la pratique, ainsi que sur le plan des mentalités.

Au niveau du droit positif, les réformes effectuées n'étant pas limitées par le texte constitutionnel, il s'agit également de l'internationalisation du droit des Droits de l'homme ou bien à l'inverse, il s'agit plutôt de la nationalisation du droit international des Droits de l'homme. La liberté d'expression se trouve au cour de cette interaction réciproque. La révision législative qui l'a accompagnée n'est pas non plus négligeable.

Quant à la pratique, l'avancée linéaire de la jurisprudence est constante. Les juges de première instance et des cours suprêmes s'approprient au fur et à mesure la $\mathrm{CEDH}$ et les arrêts de la Cour EDH. Ils se réferent également aux instruments internationaux des Droits de l'homme autres que la $\mathrm{CEDH}$, tels que les «pactes jumeaux» : le Pacte international relatif aux droits civils et politiques et le Pacte international relatif aux droits économiques, sociaux et culturels ${ }^{96}$.

Enfin, il nous est possible d'évoquer des mutations auxquelles nous assistons sur le plan des mentalités. Par l'évolution des mentalités, les thèmes considérés autrefois comme tabous se trouvent désormais au cour des débats consacrés à la liberté d'expression. Les discussions vives et intenses dans les médias et au sein d'organisations de la société civile signifient bien l'ouverture sur le plan des idées ${ }^{97}$.

Malgré tout, les progrès constatés en ce qui concerne le droit positif et la pratique ainsi que sur le plan des mentalités ne semblent pas suffisants. Premièrement, le processus de la révision législative en ce qui concerne la liberté d'expression reste insuffisant ${ }^{98}$. Les lois fondamentales relatives à la liberté d'expression doivent être renouvelées ou bien révisées : la loi sur les partis politiques, le Code pénal, la loi de la presse et la loi de lutte contre le terrorisme sont, parmi d'autres choses, susceptibles de créer le délit d'opinion. Certes, l'élimination du délit d'opinion nécessite aussi la révision de la Constitution qui ne manque pas de dispositions menaçant la liberté d'expression ${ }^{99}$.

Ensuite, au sein des instances juridictionnelles, une certaine résistance face aux réformes relatives aux Droits de l'homme ne se manifeste pas forcément contre les progrès réalisés. Elle est due davantage aux tendances personnelles plutôt nationalistes, souverainistes ou étatistes $^{100}$.

Il manque indéniablement une volonté politique permettant une mobilisation générale favorable au respect de la liberté d'opinion.

95. Ü. Kılınç, La Liberté d'expression..., p. 364.

96. La Turquie a ratifié avec beaucoup de retard ces deux pactes en 2003.

97. Le projet lancé par le Gouvernement de l'AKP (Parti de la Justice et du Développement) en été 20o9, sous le slogan «l'ouverture kurde» ou «l'ouverture démocratique ", a créé une atmosphère propice aux débats d’idées sur les multiples problèmes concernant le pays. Pour ce qui concerne les minorités en Turquie, voir I. Ö. Kaboğlu, «La Turquie à l'épreuve des droits des minorités et des droits culturels: du traité de Lausanne aux instruments du Conseil de l'Europe", in Statut et Protection des minorités: exemples en Europe occidentale et centrale ainsi que dans les pays méditerranéens, L. Trócsányī et L. Congnard (dir.), Bruxelles, Bruylant, 2009, p. 233-265.

98. «Le Rapport annuel de Bianet sur la liberté d'expression a relevé qu'en 2009, 323 personnes ont été poursuivies en violation de la liberté d'expression, dont 123 journalistes. 101 personnes ont été condamnées à un total de 98 années de prison. 9 périodiques ont été suspendus. 36 journalistes ont été emprisonnés. » Amnesty International, Revue de presse sur la Turquie, février 2010, p. 9.

99. Plusieurs initiatives civiles dans le processus de renouvellement de la Constitution ont déjà tracé les critères de restrictions de la liberté d'expression comme une sorte de dénominateur commun tels que l'appel à la violence, le racisme et l'intolérance religieuse.

100. Pour les procès en cours, voir Antenna bulletin hebdomadaire pour la liberté d'opinion, recueilli et publié par l'Initiative contre le délit d'opinion: Düşün, düşün... Haftalık Düşünce Özgürlüğü Bülteni BIANET (Réseau indépendant de communication) qui effectue les publications périodiques sur l'Internet (www.bianet.org, www.antenna-tr.org) dans le domaine des violations des Droits de l'homme et surtout de la liberté d'expression. 
